\title{
UNA RELAZIONE DIRETTA \\ FRA LA VELOCITÀ DELLE ONDE ELASTICHE E LA DENSITÀ NELL'INTERNO DELLA TERRA
}

\author{
Paolo Emilio Valle
}

Introduzione. - Le principali equazioni di stato, che possono portare un contributo alla soluzione dei problemi relativi alla costituzione interna della Terra, derivano dal metodo Thomas-Fermi, dalla teoria delle deformazioni finite di Murnaghan, e dalla teoria dei solidi, sviluppata sulla base delle idee di Einstein e Debve.

Il metodo Thomas-Fermi è stato applicato da vari autori, tra $i$ quali Jensen ( ${ }^{1}$, che ha fornito, per il ferro, un diagramma densitàpressione di particolare interesse per le ricerche sulla costituzione del nucleo terrestre. Diagrammi dello stesso tipo sono stati più recentemente costruiti anche da Elsasser $\left({ }^{2}\right)$.

Ma le pressioni che si verificano nell'interno della Terra, non sembrano essere tanto elevate da consentire l'applicazione del metodo, il quale trascura la struttura dell'atomo e considera i suoi elettroni come un gas che obbedisca alla statistica di Fermi. Anche se si considerano le parti più interne della Terra, come lo strato $E$ del nucleo, i risultati forniti dal metodo lasciano un largo margine d'incertezza.

La teoria delle deformazioni finite di Murnaghan $\left({ }^{3}\right)$ è costruita su basi termodinamiche e si presta bene allo studio dei materiali sotto alte pressioni.

Birch $\left({ }^{4-5}\right)$ però osserva che la possibilità di valutare le velocità delle onde elastiche, in materiali sotto alte pressioni, è limitata dal fatto che i termini di ordine superiore al secondo, che appaiono nella espressione della energia di deformazione, non sono conosciuti. Peraltro Birch, partendo dall'ipotesi che l'energia libera di Helmholtz sia una funzione della deformazione, esprimibile mediante uno sviluppo in serie $\mathrm{i}$ cui coefficienti dipendano dalla sola temperatura, ha dedotto una semplice ed utile equazione di stato per materiali isotropi ed omogenei sottoposti a pressioni idrostatiche.

Il confronto con le misure eseguite da Bridgman $\left({ }^{6}\right)$ sulla variazione di volume dei metalli alcalini, fino a pressioni di $10^{5}$ atmosfere, 
ha mostrato che l'equazione di stato dedotta da Birch si accorda bene con l'esperienza, tenendo conto dei soli termini del secondo ordine.

La teoria dei solidi è stata sviluppata, sulle basi date da Einstein e Debye, da diversi autori, fra i quali Born, che ha elaborato una teoria rigorosa ( $\left.{ }^{7}\right)$ per le sostanze cbe si trovino ad una temperatura superiore alla temperatura di Debye.

Ma la possibilità di utilizzare i risultati di Born, a scopi geofisici, è limitata soprattutto dalla particolare scelta del tipo di forze che si esercitano fra le particelle. Un'applicazione semiqualitativa della teoria dei solidi all'interno della Terra è stata fatta da Ramsey $\left({ }^{8}\right)$, per discutere l'ipotesi compressibilità-pressione $\left({ }^{9}\right)$ avanzata da Bullen, mentre un'applicazione quantitativa è stata eseguita da Rikitake $\left({ }^{10}\right)$ per valutare la variazione della conducibilità elettrica con la pressione.

In questa nota viene mostrato come sia possibile dedurre, sotto certe condizioni, un'utile relazione fra la velocità delle onde elastiche di un materiale isotropo ed omogeneo e la sua densità, partendo dall'equazione di stato della teoria dei solidi, ma evitando di introdurre ipotesi particolari sul tipo di forze che si esercitano fra le particelle.

Il confronto della teoria con i dati forniti dalla geofisica risul!a soddisfacente.

Teoria. - Le pressioni interne mediante le quali un solido omogeneo equilibra una pressione esterna $p$ di tipo idrostatico, sono dovute agli sforzi statici e all'agitazione termica $\left({ }^{11 \cdot 14}\right)$. La valutazione della pressione dovuta agli sforzi statici, richiede la conoscenza delle forze che si esercitano fra le particelle del solido, mentre la pressione di agitazione termica può essere identificata con la pressione di radiazione di un sistema di onde elastiche longitudinali e trasversali completamente diffuse nel solido stesso. La lunghezza di queste onde non può essere superiore al doppio della distanza tra due particelle e quindi lo spettro delle frequenze è limitato superioramente.

Se si considera un solido di tipo monoatomico, isotropo, e si indicano con gli indici $l$ e $t$ le grandezze che si riferiscono rispettivamente alle onde longitudinali e trasversali, le frequenze massime sono date dalla relazione

$$
v_{\mathrm{m}}=v_{\mathrm{m}}\left(\frac{3 N}{4 \pi \boldsymbol{V}}\right)^{1 / 3} \quad(\mathrm{~m}=l, t)
$$

dove $v_{m}$ è la velocità, di cui verrà trascurata la dipendenza dalla 
lunghezza d'onda, ed $N$ il numero di atomi contenuti nel volume $V$, che nel seguito verrà considerato come il volume specifico dello sostanza, cioè il reciproco della densità $\varrho$. Le temperature di Debye $v_{m}$ sono due: l'una relativa alle onde longitudinali, l'altra alle onde trasversali e si ha

$$
v_{\mathrm{m}}=\frac{h v_{\mathrm{m}}}{k} \quad(m=l, t)
$$

nella quale $h$ è la costante di Plank e $k$ la costante di Boltzmann.

In una precedente nota $\left({ }^{15}\right)$ è stato mostrato che, se la temperatura è superiore, anche di poco, alla temperatura di Debye $v_{l}$, la pressione dovuta all'agitazione termica può essere scritta nella forma

$$
p_{1}=3 N k T_{\varrho} \frac{\partial \log \left(\varrho v_{1} v^{2}\right)^{1} / s}{\partial \log \varrho}
$$

e quindi l'equazione di stato risulta

$$
p=f(\varrho)+C_{\mathrm{v}} T_{\varrho} \gamma
$$

in cui $f(\varrho)$ rappresenta la pressione dovuta agli sforzi statici, $C_{v}$ il calore specifico a volume costante e si è posto

$$
\gamma=\frac{\partial \log \left(\mathrm{Q} v_{1} v_{\mathrm{t}}^{2}\right)^{1} / 3}{\partial \log \varrho}
$$

Si osservi che le velocità $v_{1}$ e $v_{\mathrm{t}}$ derivano dai moduli di elasticità isotermi, in quanto il solido è in equilibrio termico.

Indicando ora con $a$ la dilatazione termica. e con $K_{\mathrm{T}}$ l'incompressibilità isoterma, si ha dalla relazione termodinamica

$$
\left(\frac{\partial}{\partial T}\right)_{\mathrm{e}}=\alpha K_{\mathrm{T}}
$$

e dalla equazione di stato [4]

$$
\frac{\alpha K_{\mathrm{T}}}{\overline{\mathrm{c}}_{\mathrm{v}}}=\varrho \gamma+\varrho T\left(\frac{\partial \gamma}{\partial T}\right)_{\rho}
$$

Nella nota precedente si è implicitamente ammesso che risultati $\left(\partial \gamma / \partial_{T}\right)_{0}=0$, ma qui verrà fatta l'ipotesi esplicita che $\mathbf{i}$ moduli di elasticità, che determinano le velocità delle onde di agitazione termica, dipendano solo dalla distanza media tra le particelle, ossia dipendano dal solo volume. Con questa ipotesi, la quale appare ragionevole, tenuto anche conto delle espressioni delle suddette velocità, 
che si ottengono considerando semplici reticoli cristallini $\left({ }^{11-14}\right)$, viene affermata la validità della legge di Grïneisen, come risulta dalla [6] e dall'espressione [5] del parametro $\gamma$.

D'altra parte, ricordando che

$$
\frac{K_{\mathrm{T}}}{\mathrm{e}}=v_{\mathrm{l}}^{2}-\frac{4}{3} v_{\mathrm{t}}^{2}
$$

risulta che l'incompressibilità isoterma non deve dipendere, a volume costante, dalla temperatura. Ossia

$$
\left.\mid \frac{\partial \mathrm{K}_{\mathrm{T}}}{\partial T}\right)_{\mathrm{g}}=0
$$

Ora dall'equazione di stato si ha

$$
\frac{K_{\mathbf{T}}}{\varrho}=\left(\frac{\partial p}{\partial \varrho)_{\mathrm{T}}}\right)_{=}=\frac{\partial f}{\partial \varrho}+C \cdot T \frac{\partial(\gamma \varrho)}{\partial \varrho}
$$

e quindi per la [8]

$$
\frac{\partial(\gamma \rho)}{\partial \rho}=0
$$

o anche

$$
\gamma \varrho=\text { costante }
$$

Giova avvertire che la variazione dell'incompressibilità isoterma con la temperatura, a pressione costante, è controllata dalla dilatazione termica. Si ha infatti

$$
\left(\frac{\partial K_{\mathrm{T}}}{\partial T}\right)_{\mathrm{P}}=\left(\frac{\partial K_{\mathrm{T}}}{\partial \mathrm{Q}}\right)_{\mathrm{T}}\left(\frac{\partial \mathrm{Q}}{\partial T}\right)_{\mathrm{P}}+\left(\frac{\partial K_{\mathrm{T}}}{\partial T}\right)_{\mathrm{Q}}
$$

da cui, tenendo conto della [8] si ottiene

$$
\left(\frac{\partial K_{T}}{\partial T}\right)_{\mathbf{P}}=-\alpha \rho\left(\frac{\partial K_{\mathrm{T}}}{\partial \rho / T}\right.
$$

Dalla [9] consegue che la pressione dovuta all'agitazione termica dipende soltanto dalla temperatura, mentre la dilatazione termica è inversamente proporzionale alla incompressibilità e quindi l'entropia, a temperatura costante, cresce linearmente col volume, come risulta subito dalla relazione

$$
\left(\frac{\partial S}{\partial V}\right)_{\mathrm{T}}=\alpha K_{\mathrm{T}}
$$


Tali conseguenze della [9] sembrano essere piuttosto ragionevoli a temperature superiori alla temperatura di Debye.

Dalla [9], sostituendo al posto di $\gamma$ la sua espressione [5], si ottiene

$$
\varrho \frac{d \log \left(0 v_{1} v_{\mathrm{t}}^{2}\right)^{1} / 3}{d \log \varrho}=\text { costante }
$$

la quale integrata fornisce subito

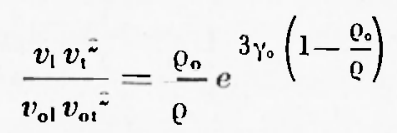

Questa relazione lega alla densità, le velocità delle onde elastiche di un materiale isotropo ed omogeneo di tipo monoatomico, che si trovi ad una temperatura superiore alla temperatura di Debye, e quindi si presenta di particolare interesse per le applicazioni geofisiche.

Si noti che dalla [10] si può ricavare la media geometrica delle frequenze massime di un solido, in funzione della densità. Infatti ricordando la [1] si ottiene

$$
\left(\frac{v_{1} v_{t}^{2}}{v_{0 l} v_{o t}}\right)^{1 / 3}=e^{\gamma_{0}\left(1-\frac{Q_{c}}{e}\right)}
$$

E inoltre possibile dare un'espressione definitiva alla legge delle trasformazioni adiabatiche reversibili. Nella nota $\left({ }^{15}\right)$ si era ottenuto per tali trasformazioni

$$
\frac{T^{3}}{\varrho v_{1} v_{\mathrm{t}}^{\bar{*}}}=\text { costante }
$$

e quindi, tenuto conto della $[10]$, si ha

$$
\frac{T}{T_{\mathrm{o}}}=e^{\gamma_{\circ}\left(1-\frac{\mathrm{\varrho}}{\mathrm{e}}\right)}
$$

o anche

$$
\frac{T}{T_{\mathrm{o}}}=e^{\gamma_{0} \frac{\Delta V}{V_{0}}}
$$

con evidente significato dei simboli.

L'esame della [10] risulta più agevole prendendo delle variabili normalizzate, ossia 


$$
\begin{gathered}
x=\frac{\varrho}{3 \gamma_{0} \varrho_{0}} \\
y=3 \gamma_{0} \frac{v_{1} v_{\mathrm{t}}^{2}}{v_{01} v_{0 \mathrm{t}}^{2}} e^{-3 \gamma_{0}}
\end{gathered}
$$

le quali risultano legate dalla relazione

$$
y=\frac{1}{x} e^{-\frac{1}{x}}
$$

Il diagramma della fig. 1 mostra l'andamento di tale funzione. Essa ammette per un massimo per $x=1$, ossia per $\varrho=3 \gamma_{0} \varrho_{0}$. Se ne deduce che, se la pressione può essere aumentata, senza che la struttura del solido subisca variazioni, fino a far assumere alla densità un valore che può valutarsi da 3 a 6 volte il valore della densità a pressione nulla, dato che $\gamma_{0}$ a tale pressione è compreso, per molti materiali, tra 1 e 2 , la velocità delle onde elastiche comincia a dedecrescere per un ulteriore aumento della densità.

È dubbio tuttavia che un materiale possa raggiungere tale stato.

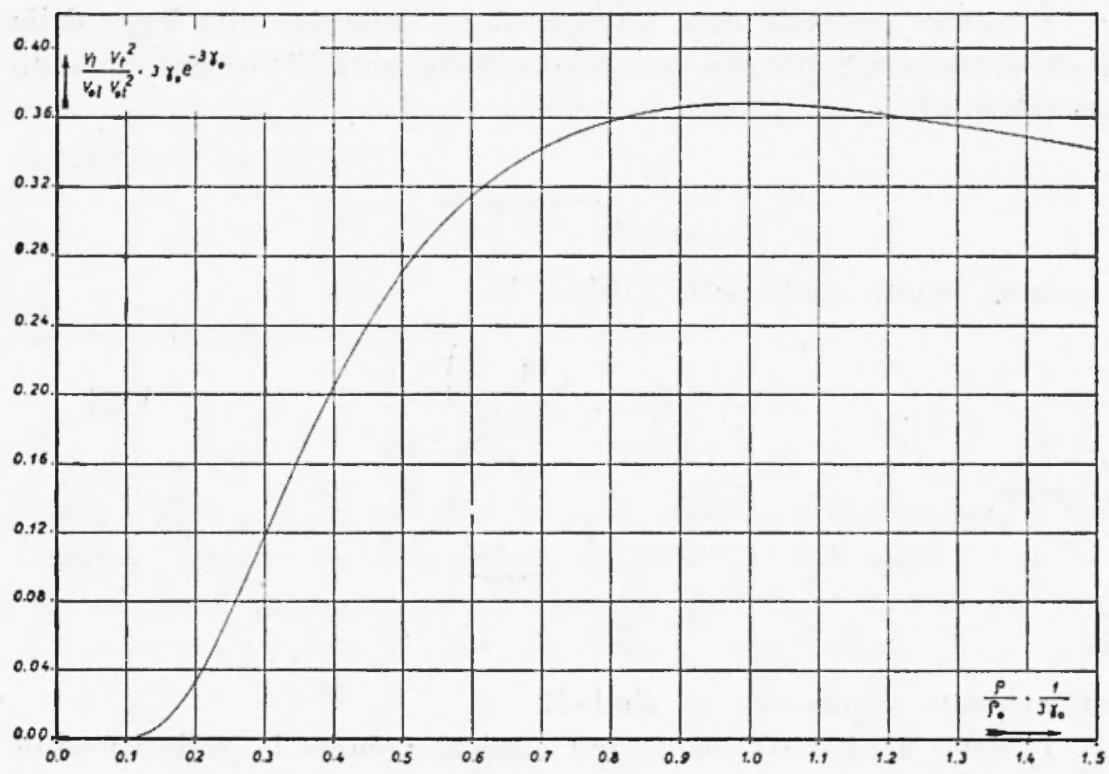

Fig. 1 
Successive e più o meno drastiche transizioni di fase potrebbero avvenire, variando i parametri relativi alle condizioni iniziali.

Applicabilità della teoria all'interno della Terra. - Le velocità delle onde elastiche contenute nella [10] sono velocità isoterme, mentre la geofisica fornisce le velocità adiabatiche. Ma si può vedere che è possibile sostituire alle velocità isoterme le velocità adiabatiche, senza che si commetta un errore troppo sensibile.

$\mathrm{Si}$ osservi che la rigidità isoterma è identica alla rigidità adiabatica e quindi nessun errore viene commesso per le onde trasversali. Per quanto riguarda le onde longitudinali, si può valutare il divario tra le due velocità, utilizzando la relazione.

$$
\frac{K_{\mathrm{s}}}{K_{\mathrm{T}}}=\frac{C_{\mathrm{p}}}{C_{\mathrm{v}}}=1+\alpha \gamma T
$$

nella quale $K_{\mathrm{s}}$ è l'incompressibilità adiabatica e $C_{\mathrm{p}}$ il calore specifico a pressione costante.

Tenuto conto che risulta

Si ottiene

$$
\begin{aligned}
& \frac{K_{\mathrm{T}}}{\varrho}=\left(v_{1}^{2}\right)_{\mathrm{T}}-\frac{4}{3} v_{\mathrm{t}}^{2} \\
& \frac{K_{\mathrm{S}}}{\varrho}=\left(v_{1}^{2}\right)_{\mathrm{S}}-\frac{4}{3} v_{\mathrm{t}}^{2}
\end{aligned}
$$

$$
\left(v_{1}^{2}\right)_{\mathrm{S}}=\left(v_{1}^{2}\right)_{\mathrm{T}}+\alpha \gamma T\left[\left(v_{1}^{2}\right)_{\mathrm{T}}-\frac{4}{3} v_{\mathrm{t}}^{2}\right]
$$

Ricordando poi che $\left(v_{1}\right)_{S} \simeq v_{\mathrm{l}} \bar{\gamma} \overline{3}$ si ha

$$
\left(v_{1}\right)_{\mathrm{S}} \simeq\left(v_{1}\right)_{\mathrm{T}}\left(1+\frac{5}{18} \alpha_{\gamma} T\right)
$$

Ora $\gamma$ vale in media nel mantello $\sim 1,5$ ed $\alpha$ è dell'ordine di $10^{-5}$, quindi

$$
\left(v_{1}\right)_{\mathrm{S}} \simeq\left(v_{1}\right)_{\mathrm{T}}\left(1+0,410^{-5} T\right)
$$

Assumendo come temperatura media nel mantello, una temperatura compresa tra $\mathrm{i}$ tre e $\mathrm{i}$ quattromila gradi, l'errore non supererà in media l'1\% o il $2 \%$. 
La teoria precedente riguarda un solido omogeneo, isotropo, di tipo monoatomico ed è quindi applicabile agli elementi e ai cristalli ionici di tipo particolare, mentre i materiali cbe compongono il mantello della Terra sono costituiti principalmente da silicati, la cui struttura è piuttosto complessa. Ma le misure del calore specifico eseguite da Wite (5) sin dal 1919 su numerosi silicati, mostrano cbe, a temperature dell'ordine di $1000{ }^{\circ} \mathrm{C}$, tali composti si possono considerare come semplici cristalli ionici, i cui atomi abbiano un peso atomico medio assai prossimo a 20 .

Occorre infine notare che le temperature presumibilmente esistenti nell'interno della Terra, sono superiori alla temperatura $v_{1}$ di Debye, la quale può essere valutata circa $750{ }^{\circ} \mathrm{C}$ alla sommità del mantello e a circa $1700{ }^{\circ} \mathrm{C}$ alla sua base.

Si conclude quindi che la teoria è applicabile con buona approssimazione al mantello della Terra. Non sembra inoltre che vi siano serie ragioni che ne impediscano l'applicabilita al nucleo.

Confronto con $i$ dati geofisici. - La velocità delle onde sismiche nell'interno della Terra sono state dedotte da vari autori, in base alle dromocrone sperimentali di tali onde.

Un'accurata determinazione è stata eseguita da Jeffreys (10-17), sebbene successive ricerche di Gutenberg $\left({ }^{18-19}\right)$ abbiano posto in evidenza la necessità di una certa revisione delle cifre fornite da Jeffreys per lo strato $B$ e $C$ del mantello, e le partì più interne dello strato $E$ del nucleo.

Comunque tale revisione per gli strati $B$ e $C$ non sembra ancora essere stata effettuata in modo definitivo, mentre per lo strato $E$ del nucleo il nuovo andamento della velocità delle onde longitudinali è stato fornito da Gutenberg soltanto sotto forma di un diagramma.

Qui verranno usate le velocità di Jeffreys, le quali per lo strato $E$ del nucleo sono leggermente più elevate rispetto alla valutazione di Gutenberg.

L'andamento della densità nell'interno della Terra è stato calcolato da Bullen $\left(^{20-22}\right)$ col metodo termodinamico Adams-Williamson.

Per uno strato omogeneo, in equilibrio idrostatico, la variazione della densità con la profondità è data da $\left({ }^{5}\right)$

$$
\frac{d \varrho}{d r}=-\frac{g \varrho}{v_{1}^{2}-\frac{4}{3} v_{\mathrm{t}}^{2}}\left(1-\frac{\gamma C_{\mathrm{p}} \tau}{g}\right)
$$


nella quale le velocità sono adiabatiche, $g$ rappresenta il campo gravitazionale e $\tau$ la differenza tra il gradiente di temperatura attuale e il gradiente adiabatico.

Bullen ha assunto come dati la massa e il momento d'inerzia della Terra. Inoltre, in base a varie considerazioni, ha potuto fissare il valore della densità alla profondità di $33 \mathrm{~km}$, che è risulıato $3,32 \mathrm{~g} \mathrm{~cm}^{-3}$.

In accordo con l'andamento delle velocità delle onde sismiche, lıa diviso il mantello della Terra in quattro strati. Lo strato $A$ compreso tra 0 e $33 \mathrm{~km}$, lo strato $B$, tra 33 e $413 \mathrm{~km}$, lo strato $C$, tra 413 e $1000 \mathrm{~km}$, lo strato $D$, tra 1000 e $2898 \mathrm{~km}$. Il nucleo della Terra è stato diviso in tre strati. Lo strato $E$ compreso tra 2898 e $4980 \mathrm{~km}$, lo strato $F$ tra 4980 e $5120 \mathrm{~km}$, e lo strato $G$ tra 5120 e $6370 \mathrm{~km}$.

Posto che la densità sia sempre una funzione crescente della profondità, Bullen, servendosi delle velocità di Jeffreys, ha applicato la [18] agli strati $B, D, E$, con l'ipotesi che il termine $\gamma_{\mathrm{p}^{\mathrm{T}}}^{\mathrm{T} / g}$ sia tanto piccolo da poter essere trascurato. In altre parole Bullen ha supposto che l'equilibrio negli strati $B, D$ ed $E$ sia assai prossimo all'equilibrio adiabatico. Ciò è abbastanza ragionevole se si pensa che la conducibilità termica delle roccie è molto piccola.

Poiché è necessario lasciare un largo margine d'incertezza per la densità dello strato $G$, l'andamento della densità negli altri strati non è del tutto determinato. Bullen ha mostrato che si possono fare due ipotesi estreme: $i)$ che la densità vari con continuità attraverso tutto il nucleo terrestre e cioè negli strati $E, F$ e $G$; ii) che la densità al centro della Terra sia $10 \mathrm{~g} \mathrm{~cm}^{-3}$ più grande che nel caso $i$ ). Per lo strato $B$ i valori delle densità nei due casi coincidono, negli strati $C$ e $D$ differiscono soltanto di qualche percento, mentre nello strato $E$ il divario è molto più sensibile.

Pertanto in questa nota sono stati presi in considerazione, per il mantello, i valori della densità corrispondente al caso intermedio fra i) e ii), mentre per lo strato $E$ del nucleo sono stati considerati il caso i), il caso ii) e il caso intermedio.

La tabella I contiene le densità e le velocità per il mantello e la tabella II $\left(^{*}\right)$ le analoghe grandezze per il nucleo.

(*) I valori delle velocità alla profondita di $500 \mathrm{Km}$ contenuti nella tabella I, sono un poco superiori a quelli corrispondenti e contenuti nella tabella I della nota (15). Ciò è dovuto ad una precedente poco accurata interpolazione, che peraltro non infuisce sensibilnente sui risultati della predetta nota. 


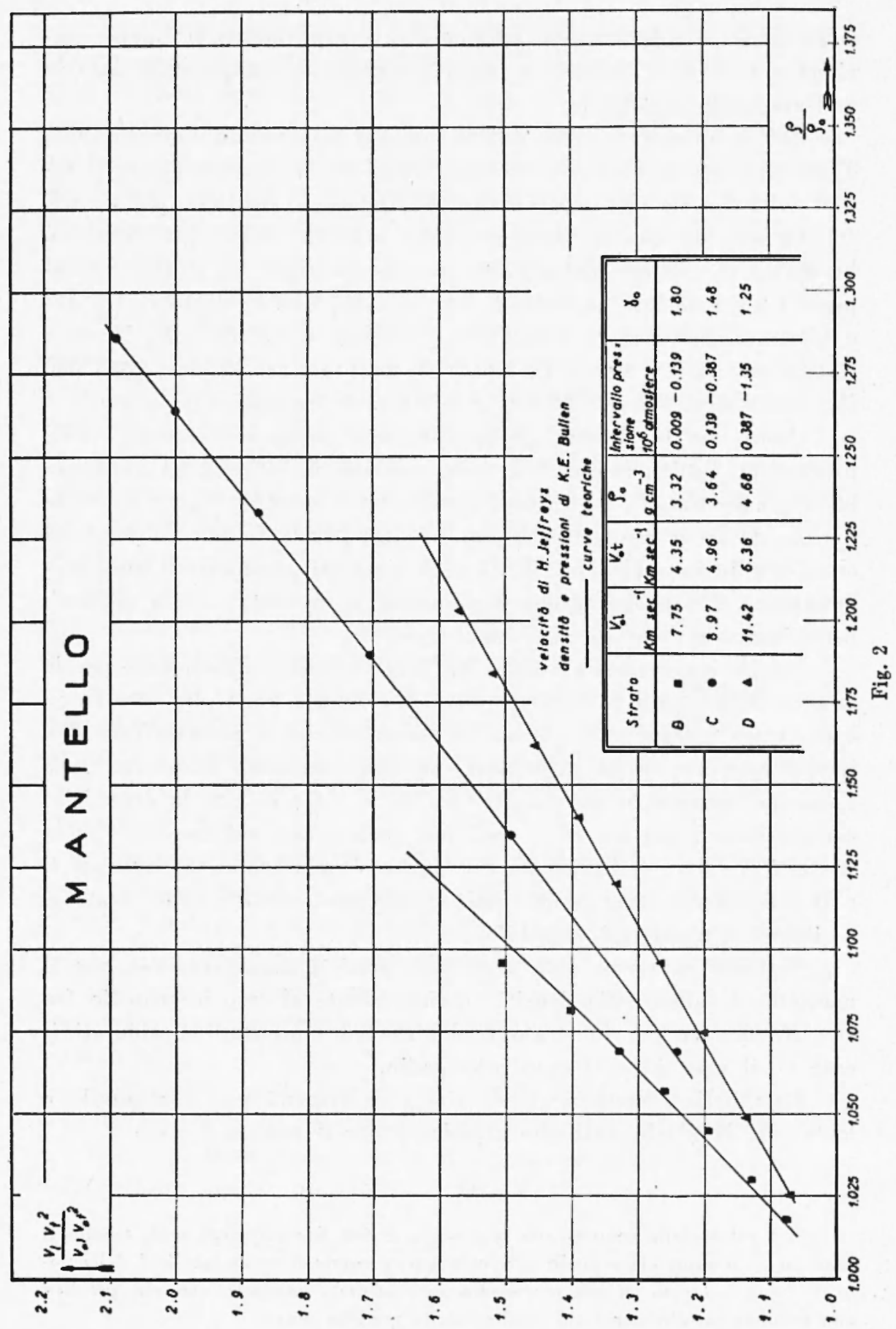




\section{TABELLA I}

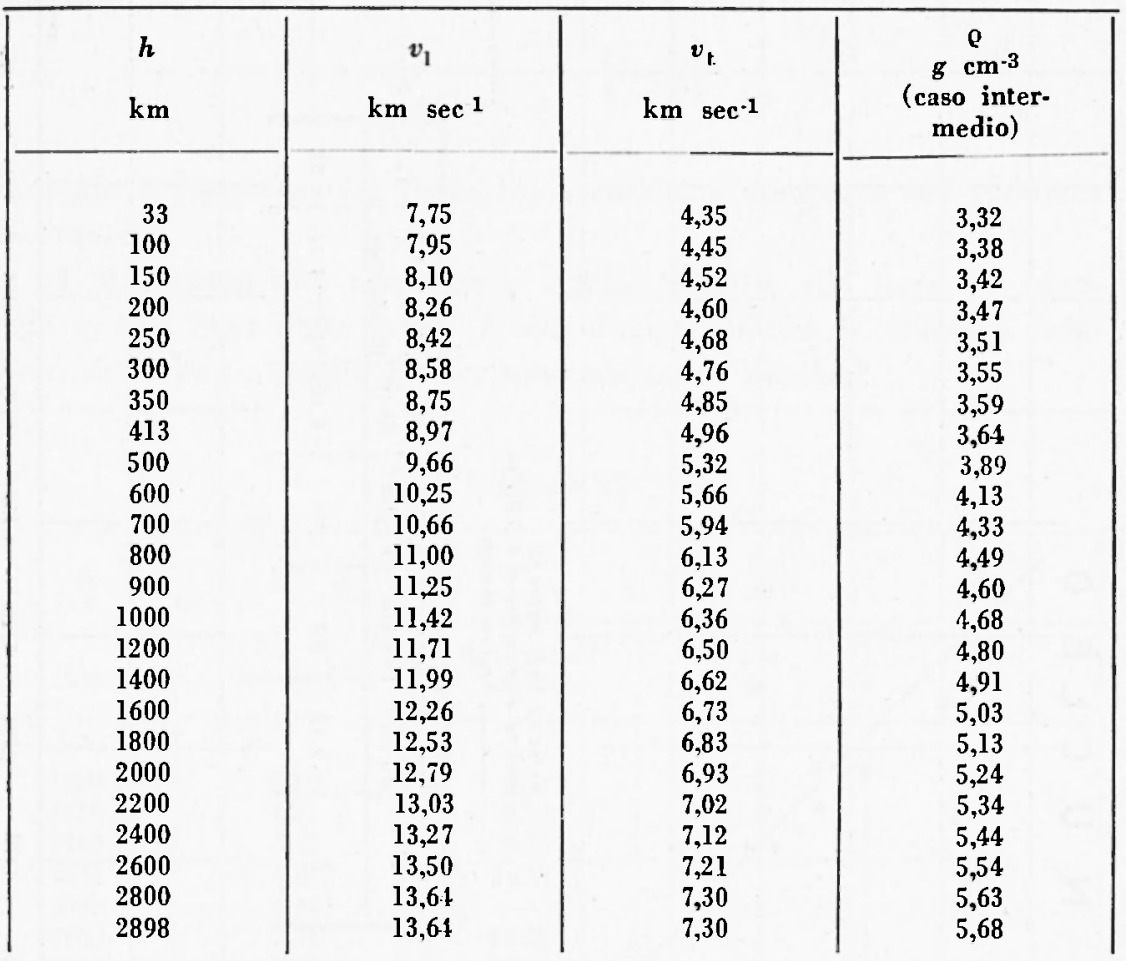

Allo scopo di effettuare un rapido confronto della teoria precedentemente sviluppata con i dati di Jeffreys e di Bullen, si è proceduto nel modo seguente. Per il mantello sono stati calcolati, per ciascuno strato, mediante le cifre contenute nella tabella $I$, i rapporti $v_{1} v_{1}^{2} / v_{\mathrm{ol}} v_{\mathrm{ot}}^{2}$ e $\mathrm{e} / \varrho_{0}$, prendendo come valori iniziali $v_{\mathrm{ol}}, v_{\mathrm{ol}}, \rho_{\mathrm{o}}$ i valori assunti da queste grandezze alla sommità di ciascuno strato. Tali rapporti sono contenuti nel diagramma della fig. 2. Per ogni strato è stato poi valutato il parametro $\gamma_{0}$, usando una coppia intermedia di detti rapporti ed è stata calcolata la funzione

$$
\frac{v_{1} v_{\mathrm{t}}^{2}}{v_{\mathrm{ol} 1} v_{\mathrm{ot}}^{2}}=\frac{\varrho^{\circ}}{\varrho} e^{3 \gamma^{\circ}\left(1-\frac{\mathrm{Q}^{\circ}}{\varrho}\right)}
$$

la quale è riportata, nel diagramma della stessa figura, come una linea continua. Per lo strato $E$ del nucleo sono stati calcolati, in base alle cifre contenute nella tabella II, i rapporti $v_{1}^{3} / v_{0}{ }^{3}$ e $\mathrm{e} /(\mathrm{c}$ os i quali sono contenuti nel diagramma della fig. 3 . 


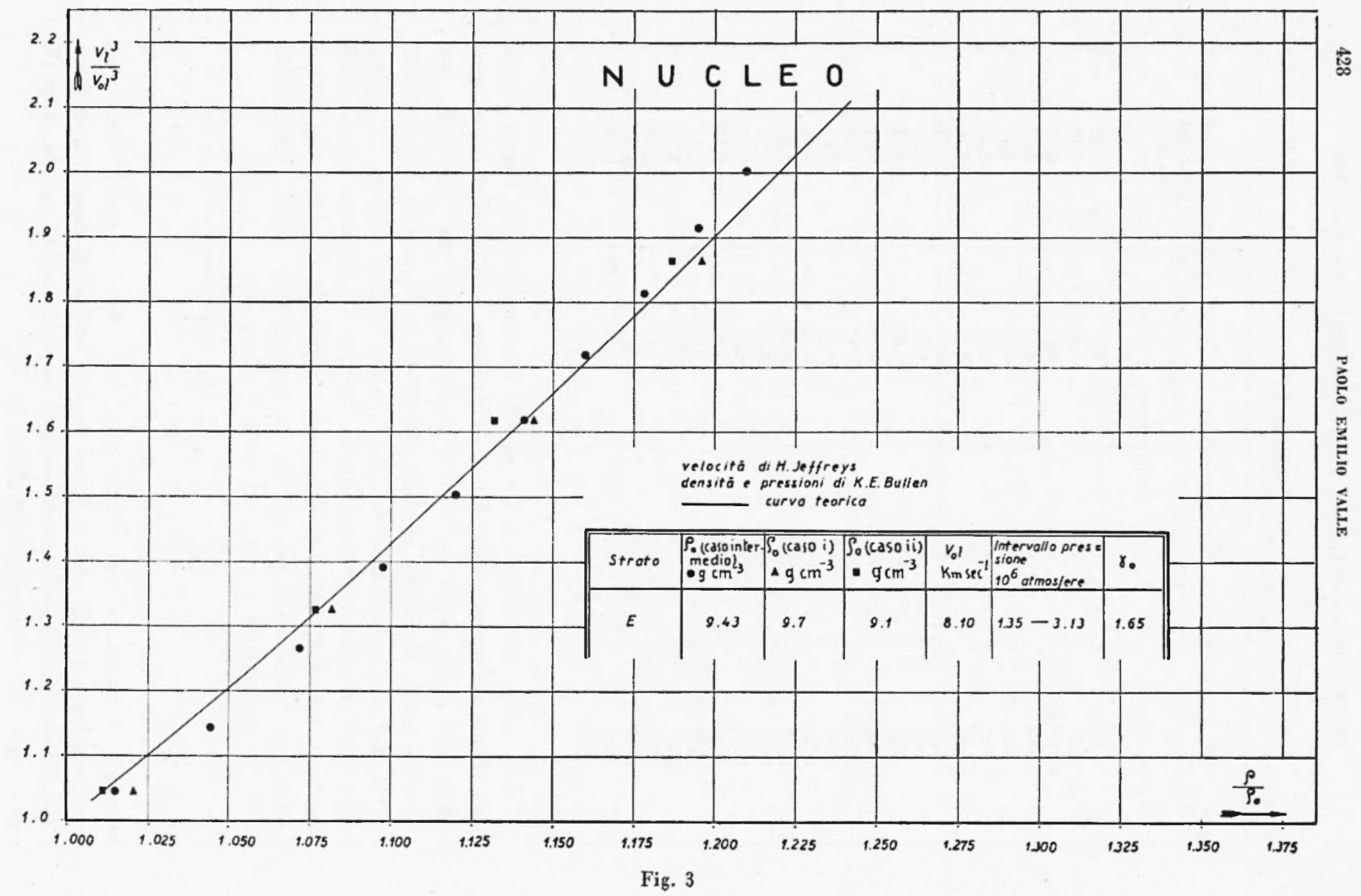


In modo apalogo al precedente s stato valutato $\gamma_{0}$ ed $\dot{e}$ stata calcolata la funzione

$$
\frac{v_{1}^{3}}{v_{0 \mathrm{t}}{ }^{1}}=\frac{\varrho_{\mathrm{o}}}{\varrho} e^{3 \gamma^{\circ}\left(1-\frac{\varrho^{\circ}}{\varrho}\right)}
$$

la quale è rappresentata dalla linea continua disegnata nel suddetto diagramma.

I diagrammi non contengono i punti relativi alla base del mantello e alla base dello strato $E$ del nucleo, poiché le velocità delle onde sismiche, a queste quote, sono alquanto incerte.

TABELla II

\begin{tabular}{|c|c|c|c|c|}
\hline \multirow{2}{*}{$\begin{array}{c}h \\
\mathrm{~km}\end{array}$} & \multirow[b]{2}{*}{$\mathrm{km} \sec ^{1}$} & \multicolumn{3}{|c|}{$\stackrel{\mathrm{e}}{\mathrm{cm}} \cdot \mathbf{3}$} \\
\hline & & $\begin{array}{l}\text { caso inter- } \\
\text { medio }\end{array}$ & $\begin{array}{l}\text { caso } \\
\text { i) }\end{array}$ & $\begin{array}{l}\text { caso } \\
\text { ii) }\end{array}$ \\
\hline 2898 & 8.10 & 9.43 & 9.7 & 9.1 \\
\hline 3000 & 8.22 & 9.57 & 9.9 & 9.2 \\
\hline 3200 & 8.47 & 9.85 & - & - \\
\hline 3400 & 8.76 & 10,11 & - & - \\
\hline 3500 & 8.90 & - & 10.5 & 9.8 \\
\hline 3600 & 9.04 & 10.35 & - & - \\
\hline 3800 & 9.28 & 10.56 & - & - \\
\hline 4000 & 9.51 & 10.76 & 11.1 & 10.3 \\
\hline 4200 & 9.70 & 10.94 & - & - \\
\hline 4400 & 9.88 & 11.11 & - & - \\
\hline 4500 & 9.97 & - & 11.6 & 10.8 \\
\hline 4600 & 10.06 & 11.27 & - & - \\
\hline 4800 & 10.25 & 11.41 & - & - \\
\hline 4982 & 10.44 & 11.54 & 11.9 & 11.1 \\
\hline
\end{tabular}

L'esame delle figure 2 e 3 mostra che l'accordo della [10] e della $\left[10^{\prime}\right]$ con i dati di Jeffreys e Bullen è piuttosto soddisfacente. Si può pertanto ritenere che la teoria sviluppata in questa nota potrà essere di qualche utilità per le ricerche sulla costituzione interna della Terra, specialmente perché la [10] e la $\left[10^{\prime}\right]$ non richiedono le particolari ipotesi dell'equilibrio idrostatico e adiabatico, connesse con metodo Adams-Willianson, e dipendono dal materiale attraverso il solo parametro $\gamma_{0}$.

Roma - Istituto Nazionale di Geofisica - Settembre 1952. 


\section{RIASSUNTO}

Viene stabilita, per via teorica, una relazione diretta tra la velocità delle onde elastiche e la densità di un solido isotropo ed omogeneo, di tipo monoatomico, che si trovi ad una temperatura superiore alla temperatura di Debye.

La teoria si basa sull'equazione di stato dei solidi e sulla ipotesi che $i$ moduli di elasticitá, che determinano le velocità delle onde di agitazione termica, dipendano soltanto dalla distanza media tra le particelle del solido.

Viene discussa l'applicabilità della relazione all'interno della Ter$r a, e$ si istituisce un confronto con le velocità delle onde sismiche fornite da Jeffreys e le densità di Bullen. L'accordo tra la teoria e $i$ dati geofisici risulta soddisfacente, sia per il mantello della Terra, che per il nucleo, a cui la teoria può essere estesa.

\section{SUMMARY}

A direct relation between the velocity of elastic waves and the density of a homogenous isotropic solid has been found by theoretical treatment of the problem. The solid is supposed monoatomic and the theory applies to temperatures greater than the Debye temperature.

The theory is based on the solid state equation and on the hypothesis that the modulus of elasticity, which determines the velocities of the waves of thermal motion, depend only on the mean distance between the particles of the solid.

The applicability of this relation to the interior of the Earth is discussed and a comparison made between the velocity of sismic waves given by Jeffreys and the density of Bullen. The agreement between the theory and geophysical data is satisfactory, either for the mantle of the Earth, or for the core, to which the theory can be extended.

\section{BIBLIOGRAFIA}

(1) JENSEN H., Das Druck-Dichte Diagramm der Elemente bei höheren Drucken am Temperaturnullpunkt. Zs. Physik, 111, 373-385 (1938).

(2) Eusasser W. H., Quantum theoretical desinties of solids at extreme compres. sion. Science, 113, 105.107 (1951). 
(3) Munnaghax F. D., Finite deformations of an elastic solid. Am. J. Matl., 59. 235.260 (1937).

(4) BInch F., The variation of seismic velocities within a simplified Earth model in accordance with the theory of finite strain. Bull. Seism. Soc. Am., 29, 463-479, (1939).

(.) Bıncr F., Elasticity and constitution of Earth's interior. J. Geophys. Res, $57,227.286$ (1952).

(6) Bridginan P. W., The compression of 39 substances to $100.000 \mathrm{~kg} / \mathrm{cm}^{2}$. Proc. Am. Acad. Arts Sci, 76, 55-70 (1948).

(7) Bons M., Thermodynamics of crystals and melting. J. Chem. Phys., 7, 591.603 (1939).

(s) Rausey W. H.. On the compressibility of the Earth. M.N.R.A.S. Geophys. Suppl. 6, $42-49$ (1950).

(9) Bullen K. E, Compressibility - pressure hypothesis and the Earth's interior. M.N.R.A.S., Geophys. Suppl. 5, 355-368 (1949).

(10) Rikitake T., Electrical conductivity and temperature in the Earth. Bull. Earth. Res. Inst. 30, 13-24 (1952).

(11) Bnillouin L., Tenseurs en Mécanique et en Elasticite. Masson, Paris (1946).

(12) Bnillouin L., Wave propagation in periodic structures. Mc. Graw.Hill, New York (1946).

(1:3) Stater J. C., Introduction to Chemical Physics. Me. Graw-Hill. New York (1939).

(14) SE.tTz F., Modern Theory of Solids. Mc. Graw-Hill. New York (1940).

(15) Valle P. E., Sul gradiente adiabatico di temperatura nellinterno della Terra. Annali di Geofisica 5, 41-53 (1952).

(16) Jefrneys $\mathrm{H}$., The time of $\mathrm{P}, \mathrm{S}$ and SKS, and the velocities of $\mathrm{P}$ and $\mathrm{S}$. M.N.R.A.S. Geophys. Suppl. 4, 498-533 (1939).

(17) Jefrneys H., The time of the core wates (second paper). M.N.R.A.S. Geophys. Suppl. 4, 594-615 (1939).

(18) Gutendenc B. On the layer of relatively low uave velocity at a depht of about 80 kilometers. Bull. Seism. Soc. Am. 38, 121-118 (19.48).

(19) Gutendenc: B., PKKP, $P^{\prime} P^{\prime}$, and the Earth's core. Trans. Am. Geophys. Union 32, 373-390 (1951).

(-0) Bullex K. E., The problem of the Earth's density tariation. Bull. Seism. Soc. Am. 30, 235-250 (1940).

(21) Bullex K. E, The density rariation of the Farth's central core. Bull. Seism. Soc. Am. 32, 19.29 (1942).

(22) Bullex K. E., Introduction of the Theory of Seismology. Cambridge, University Press (1947). 